Military Technical College

Kobry Elkobbah,

Cairo, Egypt

May 27-29,2008

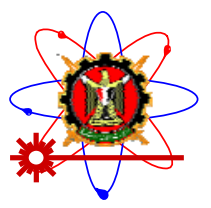

WP2

\title{
Optical Coherence Tomography
}

Ashraf El-Sherif, Yasser El-Sharkawy and Ramy Yehia

Military Technical College

\begin{abstract}
Fourier Domain optical Coherence Tomography (FD-OCT) is used to obtain subsurface cross-sectional images with micron level resolution by analyzing the interference pattern created by the mixing of light in a Michelson Interferometer FD-OCT systems are able to obtain a direct measurement of the scattering amplitude along a vertical axis within a bulk sample. One exposure provides the complete scattering profile from the surface into the bulk of the sample; this measurement is commonly referred to as an A-scan. Fourier Domain optical Coherence Tomography (FD-OCT) achieves greater sensitivity and higher imaging speed than time domain OCT and has become a powerful tool for biomedical and material application
\end{abstract}

